\section{Clinical relevance of ACOS and treatment for ACOS}

Despite GINA and GOLD recognising ACOS as a clinical reality, there is still need for more evidence on clinical phenotypes and underlying mechanisms that will help in devising a specific definition of ACOS and its treatment.

There is the possible danger of overdiagnosing ACOS, thereby overtreating COPD patients with inhaled glucocorticoids, if different definitions of ACOS are used.

Inconsistent definitions used in treatment studies make it almost impossible to determine the most effective therapy for an individual ACOS patient.

For 'easy' asthma and 'easy COPD', clear step-wise treatment approaches have been provided by GINA and GOLD, respectively. These approaches also include treatment for exacerbation reduction and comorbidities.

- 'Easy asthma': Inhaled glucocorticoids in combination with bronchodilator drugs (short- and long-acting beta-agonists (LABAs) are the main pillars. Leukotriene-receptor antagonists are an alternative choice in milder disease cases. For severe allergic asthma with appropriate IgE levels, anti-IgE treatment is an approved option.

- 'Easy COPD': The main emphasis is on smoking cessation and the use of LABAs and long-acting muscarinic antagonists (LAMAs). The role of inhaled glucocorticoids is still debatable; however, it is accepted for patients with more severe disease and those with frequent exacerbations.

- ACOS stemming from asthma: There are no firm treatment guidelines. However, due to lack of randomised clinical trials, treatment with inhaled glucocorticoids should be continued in patients with long-standing asthma, even if a component of irreversible airway obstruction develops; leukotriene modifiers may be of value in those with atopy. Combination therapy with a LAMA and a LABA is a reasonable approach for patients with more severe asthma or COPD or with overlapping conditions.

- ACOS stemming from COPD: Because of the current evidence that some COPD patients may have reversibility, eosinophilia and bronchial hyper-responsiveness, such patients may benefit from inhaled glucocorticoids.

\section{Conclusion}

With no clinical trials on ACOS, the authors believe it is still premature to recommend the designation of ACOS as a disease entity. There is need for further research to obtain a standardised definition of ACOS and its treatment; unique biomarkers will settle the debate.

\section{Ndaziona P K Banda}

Division of Pulmonology, Chris Hani Baragwanath Academic Hospital and Faculty of Health Sciences, University of the Witwatersrand, Johannesburg, South Africa

\section{References}

1. Postma DS, Rabe KF. The asthma-COPD overlap syndrome. N Engl J Med 2015;373(13):1241-1249. [http://dx.doi.org/10.1056/NEJMra1411863]

S Afr Respir J 2015;21(4):110-111. DOI:10.7196/SARJ.2015.v21i4.49

\title{
High-flow oxygen therapy in acute hypoxaemic respiratory failure
}

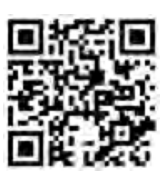

High-flow oxygen therapy allows for the delivery of heated and humidified oxygen via nasal cannula at high flow rates, thereby generating low levels of positive pressure in the upper airways. Additionally, this technique allows for the titration of the fraction of inspired oxygen as well. ${ }^{[1-3]}$ High-flow oxygen therapy has been shown to result in better comfort and oxygenation in patients with acute respiratory failure in previous studies. However, evidence for high-flow oxygen therapy on intubation rates and mortality is lacking.

Frat et al.$^{[4]}$ in a recent trial compared intubation rates within 28 days in patients who were assigned to either high-flow oxygen therapy, standard facemask oxygen therapy or non-invasive positive pressure ventilation. Secondary outcomes were the number of ventilator-free days at day 28 and all-cause mortality at 90 days. In this multicentre, open-label trial 310 patients across 23 intensive care units in Belgium and France were randomly assigned to each group. All patients included in the study had acute hypoxaemic respiratory failure without hypercapnia, and a partial pressure to fraction of respired oxygen ratio of $<300 \mathrm{~mm} \mathrm{Hg}$.

\section{Results}

The intubation rate was non-significantly different in the three groups: $38 \%$ in the high-flow oxygen group, $47 \%$ in the standard facemask oxygen group, and $50 \%$ in the non-invasive ventilation group $(p=0.18)$. The number of ventilator-free days at day 28 was analysed as a secondary outcome, and was significantly lower in the high-flow oxygen group (24 (8) days compared with 22 (10) in the facemask group and 19 (12) in the non-invasive ventilation group); for all analyses $p=0.02$. High-flow oxygen therapy was also found to significantly lower 90-day mortality as a secondary outcome compared with standard oxygen therapy and non-invasive ventilation. The hazard ratio for death at 90 days was 2.01 (95\% confidence interval (CI) 1.01 - 3.99) when comparing standard oxygen with high-flow oxygen therapy $(p=0.046)$ and $2.5(95 \% \mathrm{CI}$ 1.31 - 4.78) with non-invasive ventilation compared with high-flow oxygen ( $p=0.006$ ). Given the findings of this study, high-flow oxygen therapy appears to be a reasonable alternative to facemask oxygen and non-invasive ventilation in patients with acute hypoxaemic respiratory failure and may confer a survival benefit.

\section{Laila Suleman}

Department of Pulmonology and Critical Care, Charlotte Maxeke Johannesburg Academic Hospital and University of the Witwatersrand, Johannesburg, South Africa

\section{References}

1. Chanques G, Riboulet F, Molinari N, et al. Comparison of three high flow oxygen therapy devices: A clinical physiological cross-over study. Minerva Anestesiol 2013;79(12):1344-1355.

2. Corley A, Caruana LR, Barnett AG, Tronstad O, Fraser JF. Oxygen delivery through high-flow nasal cannulae increase end-expiratory lung volume and reduce respiratory rate in post-cardiac surgical patients. Br J Anaesth 2011;107(6):998-1004. [http:// dx.doi.org/10.1093/bja/aer265]

3. Parke RL, Eccleston ML, McGuinness SP. The effects of flow on airway pressure during nasal high-flow oxygen therapy. Respir Care 2011;56(8):1151-1155. [http://dx.doi. org/10.4187/respcare.01106]

4. Frat JP, Thille AW, Mercat A, et al. High-flow oxygen through nasal cannula in acute hypoxaemic respiratory failure. N Engl J Med 2015;372(23):2185-2196. [http://dx.doi. org/10.1056/NEJMoa1503326]

S Afr Respir J 2015;21(4):111. DOI:10.7196/SARJ.2015.v21i4.48 